\title{
Treatment of posterior circulation fusiform aneurysms
}

\author{
Ephraim W. Church, MD, ${ }^{1}$ Mark G. Bigder, MD, ${ }^{1}$ Eric S. Sussman, MD, ${ }^{1}$ \\ Santosh E. Gummidipundi, MS, ${ }^{3}$ Summer S. Han, PhD, ${ }^{1,3}$ Jeremy J. Heit, MD, PhD, ${ }^{1,2}$ \\ Huy M. Do, MD, ${ }^{1,2}$ Robert L. Dodd, MD, PhD, ${ }^{1,2}$ Michael P. Marks, MD, ${ }^{1,2}$ and \\ Gary K. Steinberg, MD, PhD'
}

Departments of ${ }^{1}$ Neurosurgery and ${ }^{2}$ Radiology, and Stanford Stroke Center; and ${ }^{3}$ Quantitative Sciences Unit, Stanford University School of Medicine, Stanford, California

OBJECTIVE Perforator arteries, the absence of an aneurysm discrete neck, and the often-extensive nature of posterior circulation fusiform aneurysms present treatment challenges. There have been advances in microsurgical and endovascular approaches, including flow diversion, and the authors sought to review these treatments in a long-term series at their neurovascular referral center.

METHODS The authors performed a retrospective chart review from 1990 to 2018. Primary outcomes were modified Rankin Scale (mRS) scores and Glasgow Outcome Scale (GOS) scores at follow-up. The authors also examined neurological complication rates. Using regression techniques, they reviewed independent and dependent variables, including presenting features, aneurysm location and size, surgical approach, and pretreatment and posttreatment thrombosis.

RESULTS Eighty-four patients met the inclusion criteria. Their mean age was 53 years, and $49(58 \%)$ were female. Forty-one (49\%) patients presented with subarachnoid hemorrhage. Aneurysms were located on the vertebral artery (VA) or posterior inferior cerebellar artery (PICA) in $50(60 \%)$ patients, basilar artery (BA) or vertebrobasilar junction (VBJ) in 22 $(26 \%)$, and posterior cerebral artery (PCA) in $12(14 \%)$. Thirty-one (37\%) patients were treated with microsurgical and 53 $(63 \%)$ with endovascular approaches. Six aneurysms were treated with endovascular flow diversion. The authors found moderate disability or better (mRS score $\leq 3$ ) in $85 \%$ of the patients at a mean 14-month follow-up. The GOS score was $\geq 4$ in $82 \%$ of the patients. The overall neurological complication rate was $12 \%$. In the regression analysis, patients with VA or PICA aneurysms had better functional outcomes than the other groups $(p<0.001)$. Endovascular strategies were associated with better outcomes for BA-VBJ aneurysms $(p<0.01)$, but microsurgery was associated with better outcomes for VA-PICA and PCA aneurysms $(p<0.05)$. There were no other significant associations between patient, aneurysm characteristics, or treatment features and neurological complications $(p>0.05)$. Patients treated with flow diversion had more complications than those who underwent other endovascular and microsurgical strategies, but the difference was not significant in regression models.

CONCLUSIONS Posterior circulation fusiform aneurysms remain a challenging aneurysm subtype, but an interdisciplinary treatment approach can result in good outcomes. While flow diversion is a useful addition to the armamentarium, traditional endovascular and microsurgical techniques continue to offer effective options.

https://thejns.org/doi/abs/10.3171/2020.4.JNS192838

KEYWORDS fusiform aneurysm; posterior circulation aneurysm; treatment strategy; vascular disorders

$\mathrm{P}$ OSTERIOR circulation fusiform aneurysms including basilar artery (BA), vertebral artery (VA), posterior inferior cerebellar artery (PICA), posterior cerebral artery (PCA), and other related aneurysms present an ongoing challenge in cerebrovascular neurosurgery. The fusiform morphology, with small but life-sustaining perforators along the brainstem, frequently precludes welldeveloped cerebrovascular and endovascular techniques such as clip reconstruction and coiling. There is a definite bleeding risk associated with vertebrobasilar fusiform aneurysms, although rates may be similar to anterior circulation aneurysms if dissecting aneurysms are excluded. ${ }^{1}$

ABBREVIATIONS BA = basilar artery; GOS = Glasgow Outcome Scale; ICA = internal carotid artery; mRS = modified Rankin Scale; PCA = posterior cerebral artery; PICA = posterior inferior cerebellar artery; $\mathrm{SAH}=$ subarachnoid hemorrhage; $\mathrm{VA}=$ vertebral artery; $\mathrm{VBJ}=$ vertebrobasilar junction.

SUBMITTED November 8, 2019. ACCEPTED April 27, 2020.

INCLUDE WHEN CITING Published online July 24, 2020; DOI: 10.3171/2020.4.JNS192838. 
However, nearly half of these aneurysms enlarge over 4 years. $^{2}$ The tiny perforators often thrombose, causing brainstem strokes. There is frequently mass effect on the brainstem that causes brain compression and obstructive hydrocephalus.

Notable microsurgical efforts in the treatment of posterior circulation fusiform aneurysms included deliberate VA or BA occlusion (Hunterian ligation). ${ }^{3}$ Bypass techniques have been developed, but success is limited, and bypass for these aneurysms has been called a "work in progress." $1,4,5$ Neuroendovascular developments include endovascular occlusion of VAs or BAs with or without bypass, coils with or without stents, and most recently flow diversion. ${ }^{6}$ The initial enthusiasm for flow diversion of posterior circulation fusiform aneurysms has been tempered by high complication rates in initial series. ${ }^{6}$ Finally, medical advances such as statins, while offering theoretical benefit, appear to have a somewhat limited impact on outcomes. ${ }^{7}$ We sought to review our experience with the treatment of posterior circulation fusiform aneurysms at Stanford University Medical Center over the last 19 years in order to track progress in the evolution of treatments for this challenging cerebrovascular condition.

\section{Methods}

We performed a retrospective cohort study of all patients with posterior circulation fusiform aneurysms treated at Stanford University Medical Center from 1990 to 2018. Patient demographic, clinical presentation, treatment, complication, and outcome details were determined from the electronic medical records. Aneurysm morphology was determined from operative reports. We recorded treatment strategy and complications. The study was approved by our institutional review board, which waived consent for study inclusion.

Multiple terms such as "dolichoectatic" or "fusiform" have been used for aneurysms not amenable to simple clipping or coiling techniques. For this study, we defined fusiform aneurysms as those with some component involving the full circumference of the vessel, making them unsuitable for straightforward clipping or coiling approaches. When treated with clipping, these aneurysms typically require complex clip reconstruction, trapping, and/or bypass. When treated with endovascular techniques, these aneurysms usually require stent-assisted coiling, parent vessel sacrifice, or vessel reconstruction with flow diversion.

The treatment strategy was formulated by cerebrovascular and endovascular experts. Cases were formally reviewed by discussion from a multidisciplinary team. Nonemergency cases were discussed in detail at our weekly interdisciplinary cerebrovascular meeting, which includes neurosurgeons and interventional neuroradiologists. The specific treatment strategy was chosen based on patient age and comorbidities, presentation including rupture status, aneurysm features, and available open cerebrovascular and endovascular options.

The coprimary outcomes were follow-up modified Rankin Scale (mRS) score and follow-up Glasgow Outcome Scale (GOS) score. A secondary outcome was neurological complications. Univariate and multivariate regression models were created to examine relationships between key independent and dependent variables, including presenting features, aneurysm location and size, surgical approach and complications, and pretreatment and posttreatment thrombosis. In a follow-up analysis, we also checked for interactions between the independent variables. For the regression analyses, we grouped VA with PICA aneurysms and BA with vertebrobasilar junction (VBJ) aneurysms, since these aneurysms share anatomical and treatment strategy similarities. Data were analyzed using Stata 15 (StataCorp) and the R Statistical Programming Language (https://www.r-project.org; R Foundation for Statistical Computing). Statistical significance was two-sided and assessed at the $\alpha=0.05$ level. The analyses were conducted in collaboration with experts in the Stanford Quantitative Sciences Unit.

\section{Results}

There were 84 aneurysms in 84 patients. The median patient age was 53 years (range 11-86 years). Forty-nine $(58 \%)$ patients were female and $35(42 \%)$ were male. Forty-one (49\%) patients presented with subarachnoid hemorrhage (SAH), 26 (31\%) with symptoms from mass effect or ischemia, and the remaining $17(20 \%)$ presented with headache or an incidentally discovered aneurysm. The median mRS and GOS scores on presentation were 3 and 4, respectively. Of the patients presenting with rupture, the median Hunt and Hess grade was III. Most (49\%) patients had Fisher grade 3 SAH. There were $34(40 \%)$ VA aneurysms, 16 (19\%) PICA aneurysms, 14 (17\%) BA aneurysms, 8 (10\%) VBJ aneurysms, and 12 (14\%) PCA aneurysms. Most (62\%) aneurysms were $\geq 10 \mathrm{~mm}$ in greatest diameter. Patient and aneurysm characteristics are presented in Table 1.

Thirty-one (37\%) aneurysms were treated with microsurgical techniques and $53(63 \%)$ with endovascular techniques. Microsurgical techniques included clip reconstruction in $11(35 \%)$, trapping usually with bypass in 8 $(26 \%)$, parent vessel occlusion in $8(26 \%)$, and wrapping in $4(13 \%)$. The most commonly used endovascular technique was parent vessel occlusion with or without balloon test occlusion in 39 (74\%) cases. Other endovascular techniques included flow diversion in $6(11 \%)$, primary coiling in 4 (7.5\%), stent coiling in $3(5.7 \%)$, and balloon-assisted coiling in $1(1.9 \%)$.

There were 12 (14\%) neurological complications overall. Three $(10 \%)$ of these occurred in the microsurgery group and $9(17 \%)$ in the endovascular group. There were $2(33 \%)$ complications in patients who underwent flow diversion. The majority $(8,67 \%)$ of neurological complications were ischemic strokes. Two of these occurred in patients with ruptured aneurysms, and it could not be determined to what extent vasospasm was responsible. There was one $(8.3 \%)$ hemorrhagic stroke and no intraoperative aneurysm ruptures. Three (25\%) neurological complications were due to progressive aneurysmal mass effect following treatment.

The overall median follow-up mRS score was 2, and the median follow-up GOS score was 4 at a mean follow- 
Church et al.

TABLE 1. Patient and aneurysm characteristics

\begin{tabular}{lcccc}
\hline \multirow{2}{*}{ Characteristic } & Overall & \multicolumn{3}{c}{ Treatment } \\
\cline { 3 - 5 } & Population & Microsurgery & Endovascular (all) & Flow Diversion \\
\hline No. of cases & 84 & $31(37 \%)$ & $53(63 \%)$ & $6(7 \%)$ \\
\hline Median age, yrs & 53 & 53 & 53 & 54 \\
\hline Female & $49(58 \%)$ & $21(68 \%)$ & $28(53 \%)$ & $5(83 \%)$ \\
\hline Presentation & & & & \\
\hline SAH & $41(49 \%)$ & $17(55 \%)$ & $24(45 \%)$ & $2(33 \%)$ \\
\hline Mass effect or ischemia & $26(31 \%)$ & $6(19 \%)$ & $20(38 \%)$ & $4(67 \%)$ \\
\hline Median presenting mRS score & 3 & 3 & 3 & 3 \\
\hline Median presenting GOS score & 4 & 4 & 4 & 4 \\
\hline Aneurysm location & & & & $3(50 \%)$ \\
\hline VA & $34(40 \%)$ & $6(19 \%)$ & $28(53 \%)$ & 0 \\
\hline PICA & $16(19 \%)$ & $10(32 \%)$ & $6(11 \%)$ & $2(33 \%)$ \\
\hline BA & $14(17 \%)$ & $4(13 \%)$ & $10(19 \%)$ & $1(17 \%)$ \\
\hline VBJ & $8(10 \%)$ & $1(3 \%)$ & $7(13 \%)$ & 0 \\
\hline PCA & $12(14 \%)$ & $10(32 \%)$ & $2(4 \%)$ & \\
\hline
\end{tabular}

up of 14 months. We found moderate disability or better (mRS score $\leq 3$ ) in $85 \%$ at a mean follow-up of 14 months. The GOS score was $\geq 4$ in $82 \%$ of the patients. Pre- and postoperative mRS and GOS scores are presented in Fig. 1. Three $(3.6 \%)$ patients died. Fourteen $(17 \%)$ patients re- quired a ventricular shunt. At the time of the last vascular imaging, 14 (17\%) aneurysms had some residual filling. Outcomes are summarized in Table 2.

Given the anatomical and treatment strategy similarities, we grouped VA with PICA aneurysms and BA with
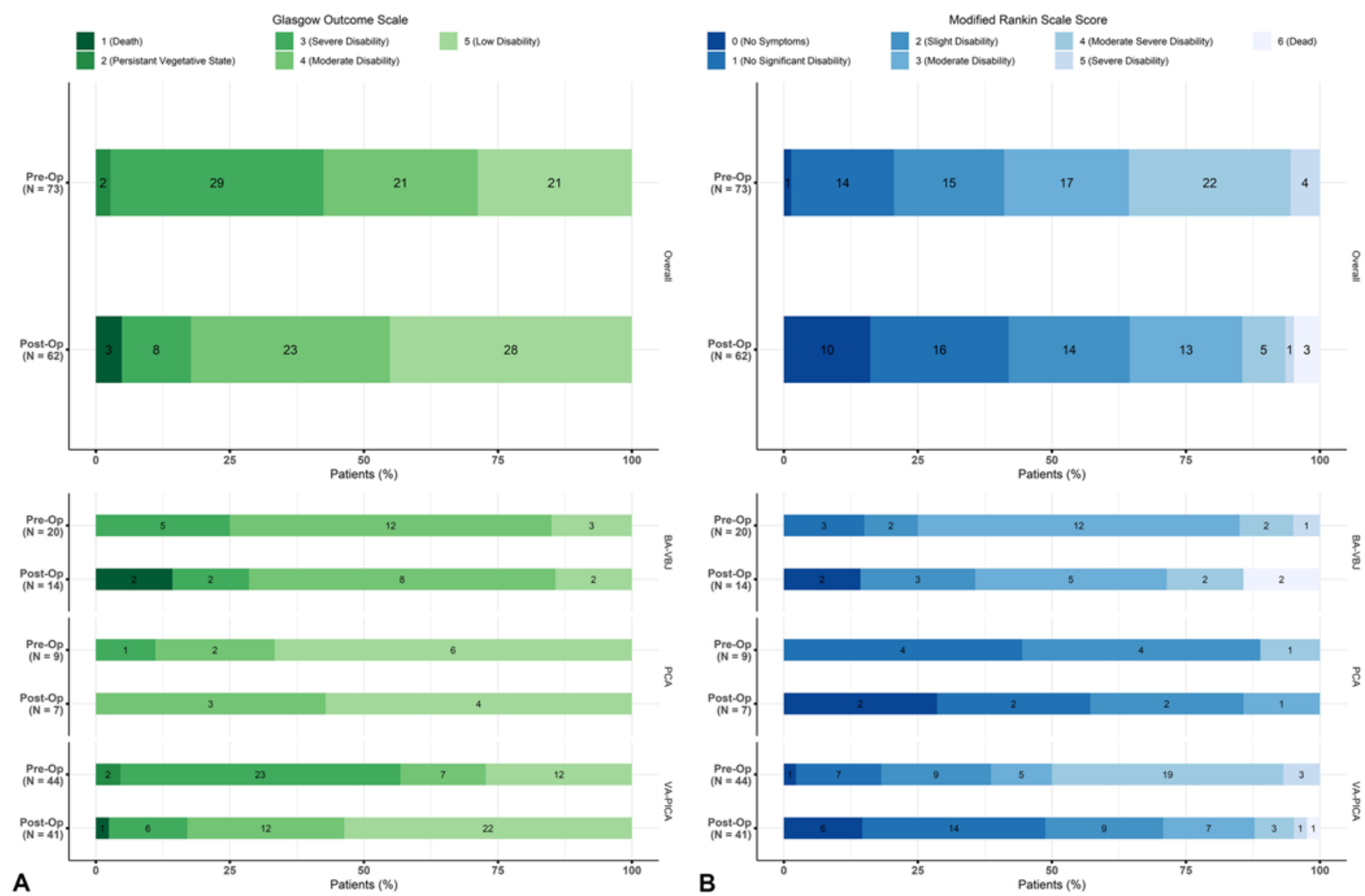

FIG. 1. Pretreatment and posttreatment GOS scores (A) and mRS scores (B). 
TABLE 2. Patient outcomes

\begin{tabular}{lccccc}
\hline & \multirow{2}{*}{$\begin{array}{c}\text { Overall } \\
\text { Outcome }\end{array}$} & \multicolumn{3}{c}{ Treatment } \\
\cline { 3 - 5 } & Population & Microsurgery & Endovascular (all) & Flow Diversion \\
\hline Median follow-up mRS score & 2 & 2 & 2 & 1.5 \\
\hline Median follow-up GOS score & 4 & 4 & 4 & 4.5 \\
\hline Neurological complication & $12(14 \%)$ & $3(10 \%)$ & $9(17 \%)$ & $2(33 \%)$ \\
\hline Ventriculoperitoneal shunt & $14(17 \%)$ & $4(13 \%)$ & $9(17 \%)$ & 0 \\
\hline Residual filling at last vascular imaging & $14(17 \%)$ & $4(13 \%)$ & $11(21 \%)$ & $1(17 \%)$ \\
\hline
\end{tabular}

VBJ aneurysms in the regression analyses. Univariate regression for follow-up mRS scores revealed a significant association between VA-PICA aneurysms and improved $\mathrm{mRS}$ scores $(\beta-1.31,95 \% \mathrm{CI}-1.99$ to $-0.63, \mathrm{p}=0.00028)$. There was a trend toward improved mRS score for PCA aneurysms $(\beta-0.87,95 \% \mathrm{CI}-1.90$ to $0.16, \mathrm{p}=0.097)$. There were no other significant associations or notable trends between patient, aneurysm, or treatment variables and follow-up mRS score, including age, $\mathrm{SAH}$, aneurysm size, partial thrombosis of the aneurysm, and treatment strategy (Table 3).

Univariate regression analysis for follow-up GOS score revealed the same findings as follow-up mRS score. There was a significant association between VA-PICA aneurysms and improved GOS score $(\beta 0.92,95 \%$ CI $0.43-1.41$, $\mathrm{p}=0.00041)$. Again, there was a trend toward improved GOS score for PCA aneurysms $(p=0.071)$. There were no other significant associations between patient, aneurysm characteristics, or treatment variables and follow-up GOS score.

The univariate regression analysis for neurological complication showed no significant associations between patient, aneurysm characteristics, or treatment variables and complications, including treatment strategy. The results are presented in Table 4.

We controlled for known confounders using multivariate techniques. Stepwise multivariate modeling was performed for follow-up mRS score and confirmed the results of univariate regression. Multivariate models for followup GOS score and neurological complications were also consistent with the univariate analysis.

We checked for interactions between the independent variables and found a meaningful interaction between surgical strategy and aneurysm location. Overall, endo-

TABLE 3. Univariate regression analysis for follow-up $\mathrm{mRS}$ score

\begin{tabular}{lccrl}
\multicolumn{1}{c}{ Variable } & $\beta$ & $2.5 \% \mathrm{Cl}$ & $97.5 \% \mathrm{Cl}$ & $\mathrm{p}$ Value \\
\hline Age & 0.01 & -0.01 & 0.03 & 0.29 \\
\hline SAH & -0.64 & -1.58 & 0.30 & 0.18 \\
\hline VA-PICA & -1.31 & -1.99 & -0.63 & 0.00028 \\
\hline PCA & -0.87 & -1.90 & 0.16 & 0.097 \\
\hline Aneurysm size & 0.02 & -0.02 & 0.06 & 0.23 \\
\hline Partial thrombosis & -0.08 & -1.11 & 0.95 & 0.88 \\
\hline Endovascular & 0 & -0.67 & 0.67 & 1.00 \\
\hline Flow diversion & 0.49 & -0.54 & 1.53 & 0.34 \\
\hline
\end{tabular}

vascular compared with microsurgical treatment strategy was associated with improved follow-up mRS and GOS scores ( $p=0.0079$ and $p=0.0084$, respectively). However, this was only true for BA-VBJ aneurysms. For VA-PICA aneurysms, microsurgery was associated with better outcomes $(\mathrm{p}=0.015$ and $\mathrm{p}=0.011)$. Microsurgery was also associated with better outcomes for PCA aneurysms ( $\mathrm{p}=$ 0.014 and $\mathrm{p}=0.025$ ).

\section{Discussion}

We present a large series of patients with treated posterior circulation fusiform aneurysms and describe overall good results in $>80 \%$ of the cases. The neurological complication rate in this series was $14 \%$. These results support the well-accepted notion that posterior circulation aneurysms remain a challenging aneurysm subtype. However, good results can be achieved when flexible decisionmaking is applied by a multidisciplinary team, whether by different specialties or dual-trained neurosurgeons.

In this series, we observed moderate disability or better (mRS score $\leq 3)$ in $85 \%$ of patients. The GOS score was $\geq$ 4 in $82 \%$ of patients at follow-up. In our initial experience with these challenging vascular lesions published in 2007, we observed GOS scores $\geq 4$ in approximately $60 \%$ of patients. ${ }^{8}$ A recent review of fusiform aneurysm series found good outcomes in $77 \%-100 \%$ of patients. ${ }^{9}$ However, there was a high degree of variability among inclusion criteria. Most studies included anterior circulation fusiform aneurysms. Among the 4 series (1-50 patients) focusing on BA aneurysms, good outcomes were seen in $66.5 \%-100 \%$ of

TABLE 4. Univariate regression analysis for neurological complication

\begin{tabular}{lcccc}
\hline \multicolumn{1}{c}{ Variable } & OR & $2.5 \% \mathrm{Cl}$ & $97.5 \% \mathrm{Cl}$ & $\mathrm{p} \mathrm{Value}$ \\
\hline Age & 1.00 & 0.96 & 1.04 & 0.93 \\
\hline SAH & 0.30 & 0.062 & 1.12 & 0.094 \\
\hline Presenting mRS score & 0.77 & 0.46 & 1.26 & 0.31 \\
\hline Presenting GOS score & 1.45 & 0.72 & 3.08 & 0.31 \\
\hline VA-PICA & 0.36 & 0.087 & 1.47 & 0.15 \\
\hline PCA & 0.70 & 0.086 & 4.15 & 0.71 \\
\hline Aneurysm size & 1.025 & 0.95 & 1.10 & 0.52 \\
\hline Partial thrombosis & 1.63 & 0.22 & 8.38 & 0.58 \\
\hline Endovascular & 1.69 & 0.45 & 8.22 & 0.46 \\
\hline Flow diversion & 2.85 & 0.36 & 16.79 & 0.26 \\
\hline
\end{tabular}


patients. Improvement in outcomes over the last decade may be due to improved techniques and increasing experience in selecting the best strategies.

Seventeen percent of aneurysms in this series had some residual filling at 14 months. We view this as a relatively low number, particularly when considering that an acceptable treatment objective for some troublesome posterior circulation fusiform aneurysms may be simply stabilization of growth and mass effect. Nevertheless, these results underscore the considerable challenge of this aneurysm type.

Not all posterior circulation fusiform aneurysms are equally challenging. In this study, patients with VA-PICA and PCA aneurysms had better outcomes compared to those with BA-VBJ aneurysms, which is consistent with what has been seen in other series. ${ }^{8,10}$ The basilar subtypes may involve perforators that can stretch and thrombose as the aneurysm grows, leading to devastating brainstem strokes. They also frequently enlarge and produce significant mass effect on the brainstem, with compression and occasionally hydrocephalus.

Some posterior circulation fusiform aneurysms may be better treated with endovascular rather than microsurgical techniques. In our series, endovascular techniques were associated with better outcomes for BA-VBJ aneurysms, while microsurgery was associated with improved outcomes for the VA-PICA and PCA aneurysms. Interestingly, SAH was not associated with worse outcomes, including disability. This speaks to the commonly progressive and injurious, albeit occasionally indolent, behavior of chronic fusiform aneurysms compared with the dissecting, ruptured type. Leibowitz and colleagues compared chronic fusiform aneurysms presenting with mass effect with fusiform aneurysms presenting with $\mathrm{SAH}$ in a series of patients undergoing endovascular parent vessel occlusion. ${ }^{11}$ The patients presenting with SAH fared significantly better than those presenting with mass effect $(\mathrm{p}<0.02)$.

Our clinical approach involves multidisciplinary review of all cases. Patients are admitted to the neurosurgery service, and every case is reviewed by open cerebrovascular, endovascular, and neurocritical care physicians. In emergency cases, frequent telephone and in-person consultations are a routine part of our practice. A weekly, interdisciplinary cerebrovascular case conference provides a collaborative forum for in-depth discussion of complex cases. Preoperative and postoperative care on the neurosurgery service provides the setting for seamless multidisciplinary patient care for these challenging cases.

\section{Flow Diversion for Posterior Circulation Fusiform Aneurysms}

Six patients were treated with flow diversion and had outcomes comparable to those in whom other techniques were used. Half of the patients had VA and the other half had BA-VBJ aneurysms. There were more complications (one medullary ischemic stroke, one death due to hemorrhage associated with dual antiplatelet agents) than other techniques, but this difference was not significant due to low numbers. An example of successful flow diversion of a vertebrobasilar aneurysm is shown in Fig. 2. A 69-yearold woman presented with gait unsteadiness due to mass

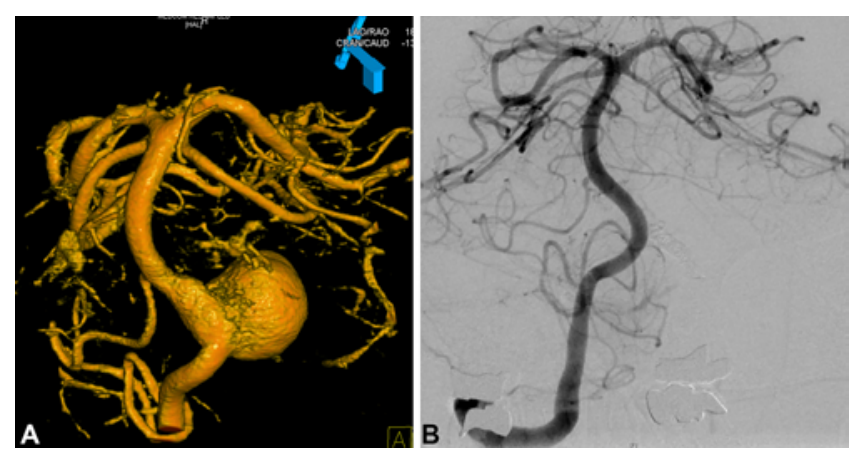

FIG. 2. Example of flow diversion embolization of a posterior circulation fusiform aneurysm. Right VA injection at treatment (A) and at 3 months posttreatment (B).

effect from a large, left-sided vertebrobasilar aneurysm. Of note, this aneurysm did include a saccular component on a fusiform dilatation involving the VBJ, and taken as a whole this was considered a fusiform aneurysm. The patient underwent flow diversion embolization of the aneurysm and placement of two flow diverters in the BA and right VA and coil embolization of the left VA. A follow-up angiogram at 3 months showed no residual aneurysm, and the patient's gait stability improved over the next several years.

The first flow diverter was FDA approved for large or giant wide-necked petrous to superior hypophyseal internal carotid artery (ICA) aneurysms in 2011 and was only available for the final 7 years $(25 \%)$ of our series. ${ }^{12} \mathrm{Al}$ though approved indications were recently expanded to include smaller aneurysms distal to the ICA bifurcation, use in the posterior circulation remains off label. ${ }^{13}$

Early enthusiasm for flow diversion of fusiform posterior circulation aneurysms has been tempered to some extent by complication rates higher than those observed in the ICA. Nevertheless, multiple series now have established flow diversion as an important tool in the treatment of posterior circulation aneurysms. ${ }^{6,14,15}$ In the largest series to date, Griessenauer and colleagues retrospectively reviewed 131 posterior circulation aneurysms treated with flow diversion at 8 academic institutions. ${ }^{6}$ Fifty-three of these were described as fusiform, and this type had the highest complication rate. There were major complications in $11.5 \%$ of the cases, and $25 \%$ of these patients had a thromboembolic complication. Still, an impressive 78\% had a follow-up mRS score of 0-2 at 11 months. A median of 2 devices were used, and nearly $60 \%$ of aneurysms were fully occluded.

Advantages of flow diversion for posterior circulation fusiform aneurysms include its ability to remodel the vessel intima, while preserving major perforators. However, perforator occlusion is sometimes observed. ${ }^{6,14,15}$ Disadvantages of flow diversion include delayed and often limited relief of severe brainstem compression and hydrocephalus, and the absolute requirement for functioning antiplatelet medication. This problem is well illustrated in a case from our own series in which an aneurysm was successfully treated with flow diversion, but the patient ultimately succumbed to significant tract hemorrhage from an external ventricular drain. 


\section{Bypass for Posterior Circulation Fusiform Aneurysms}

Open microsurgery offers unique techniques that cannot be replicated with endovascular approaches. For example, bypass surgery reroutes blood supply, allowing for aneurysm trapping and removal of mass effect when the arterial segment does not involve perforators. Parent vessel occlusion by clip placement may offer unique advantages over occlusion by coiling, because not only key branches but also tiny perforators are directly visualized. While bypass for fusiform BA-VBJ aneurysms may be considered a "work in progress,"5 VA-PICA aneurysms may be effectively treated with revascularization techniques. ${ }^{8}$

In our series, several patients with VA-PICA and PCA aneurysms were treated successfully with trapping and bypass, as illustrated in the following example cases. A 45-year-old man was found unresponsive and was transferred to an outside hospital, where the diagnosis of a ruptured, fusiform left-sided PICA aneurysm was established. He was transferred to our medical center for evaluation and treatment. A left $\mathrm{P}_{3}$ PICA-right-sided $\mathrm{P}_{3}$ PICA in situ bypass with trapping of the $1-\mathrm{cm}$ aneurysm was performed. The postoperative course was complicated by severe vasospasm requiring endovascular treatment, but he ultimately made a good recovery and was able to live independently at home (Fig. 2A and B). In a similar case, a 56-year-old woman suffered a week of severe headaches before she was found unresponsive. She was transferred to our center where the diagnosis of a ruptured, fusiform leftsided PICA aneurysm was made. She underwent a left occipital- $\mathrm{P}_{3}$ PICA extracranial-intracranial bypass and trapping of a 1-cm thin-walled, dysplastic, fusiform aneurysm. Her postoperative course was complicated by vasospasm and hydrocephalus that required the placement of a ventriculoperitoneal shunt, but ultimately she recovered and was able to live at home (Fig. 3C and D).

\section{Study Limitations}

This retrospective review is subject to several limitations. The series covers 28 years, and much changes over time. The techniques and adjuncts on both the microsurgical and endovascular fronts have advanced. Neurocritical care has become its own specialty as our understanding of the optimal pre- and postsurgical care of our most critically ill neurosurgical patients has deepened. New devices such as flow diverters offer additional options. Flow diversion was only available for the final quarter of the time period covered in this series. It is still a relatively new, albeit promising, treatment; numbers are small; and outcome data are limited.

A second, significant limitation is the heterogeneity of the included aneurysms, limiting comparisons between subgroups. For example, PICA aneurysms are commonly understood to be less challenging than BA-VBJ aneurysms, which more likely involve key brainstem perforators. To some extent, these known challenges led us to group the aneurysms as we have in this study. However, it should be recognized that each subgroup itself contains heterogeneity. For example, not all VA-PICA aneurysms are equally challenging. No two aneurysms are alike, and this should be kept in mind when interpreting these results.

Our statistical analysis is limited to known variables.

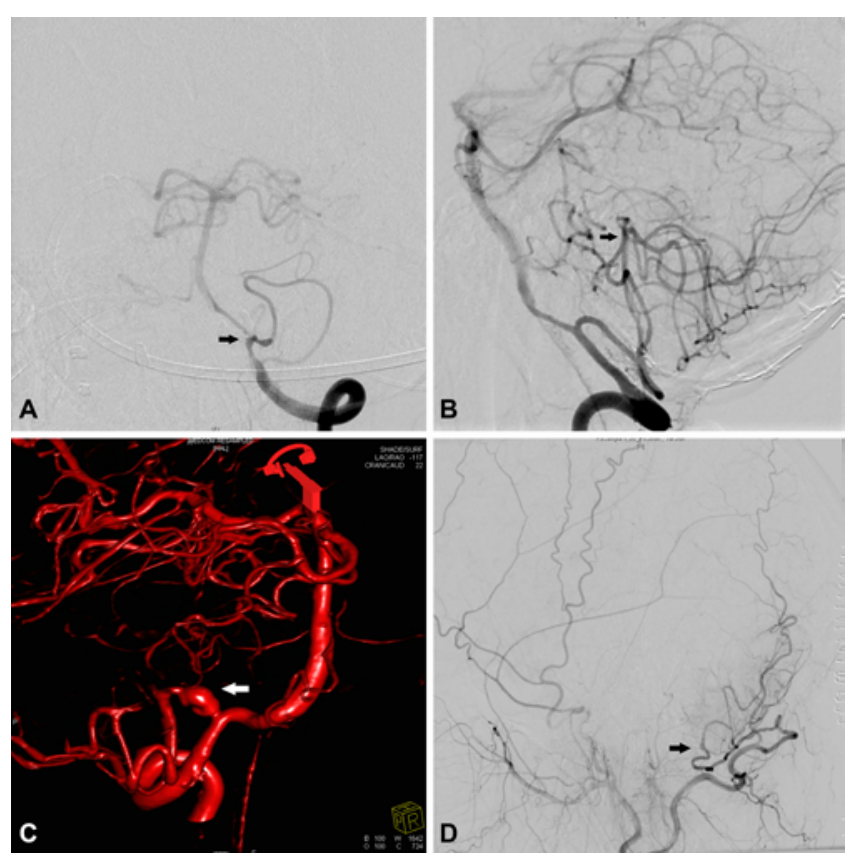

FIG. 3. Examples of common bypasses for fusiform PICA aneurysms. $A$ and B: $P_{3}$ PICA-P ${ }_{3}$ PICA in situ bypass. $C$ and D: Occipital- $P_{3}$ PICA extracranial-intracranial bypass. Arrows indicate aneurysm or bypass.

Any associations are not necessarily causal, and multiple comparisons may lead to spurious findings. However, the significant associations that we identified are clinically plausible. We did not include untreated fusiform aneurysms in this series, and we are unable to compare their outcomes. Natural history studies of these aneurysms suggest that outcomes are generally poor. Additionally, the generalizability of our findings is limited since the data are from a single neurovascular center. In the future, wellcurated neurovascular registries may provide additional answers in our quest to improve treatments and outcomes for posterior circulation fusiform aneurysms.

\section{Conclusions}

We report our experience with microsurgical and endovascular treatment of posterior circulation fusiform aneurysms at Stanford since 1990. Overall, we found good outcomes when using an interdisciplinary approach to select the best microsurgical or endovascular treatment. Flow diversion is a promising option for some fusiform aneurysms, but there are significant limitations. Microsurgical techniques such as trapping with bypass surgery remain essential components of a complete armamentarium for these challenging aneurysms.

\section{Acknowledgments}

We thank Christine Plant for editorial support.

\section{References}

1. Flemming KD, Wiebers DO, Brown RD Jr, et al. Prospective risk of hemorrhage in patients with vertebrobasilar nonsaccular intracranial aneurysm. J Neurosurg. 2004;101(1):82-87. 
2. Mangrum WI, Huston J III, Link MJ, et al. Enlarging vertebrobasilar nonsaccular intracranial aneurysms: frequency, predictors, and clinical outcome of growth. J Neurosurg. 2005;102(1):72-79.

3. Steinberg GK, Drake CG, Peerless SJ. Deliberate basilar or vertebral artery occlusion in the treatment of intracranial aneurysms. Immediate results and long-term outcome in 201 patients. J Neurosurg. 1993;79(2):161-173.

4. Evans JJ, Sekhar LN, Rak R, Stimac D. Bypass grafting and revascularization in the management of posterior circulation aneurysms. Neurosurgery. 2004;55(5):1036-1049.

5. Lawton MT, Abla AA, Rutledge WC, et al. Bypass surgery for the treatment of dolichoectatic basilar trunk aneurysms: a work in progress. Neurosurgery. 2016;79(1):83-99.

6. Griessenauer CJ, Ogilvy CS, Adeeb N, et al. Pipeline embolization of posterior circulation aneurysms: a multicenter study of 131 aneurysms. J Neurosurg. 2018;130(3):923-935.

7. Cheng W, Jia X, Li J, et al. Relationships of statin therapy and hyperlipidemia with the incidence, rupture, postrepair mortality, and all-cause mortality of abdominal aortic aneurysm and cerebral aneurysm: a meta-analysis and systematic review. J Cardiovasc Pharmacol. 2019;73(4):232-240.

8. Coert BA, Chang SD, Do HM, et al. Surgical and endovascular management of symptomatic posterior circulation fusiform aneurysms. J Neurosurg. 2007;106(5):855-865.

9. Barletta EA, Ricci RL, Silva RDG, et al. Fusiform aneurysms: a review from its pathogenesis to treatment options. Surg Neurol Int. 2018;9:189.

10. Seifert V, Raabe A, Stolke D. Management-related morbidity and mortality in unselected aneurysms of the basilar trunk and vertebrobasilar junction. Acta Neurochir (Wien). 2001; 143(4):343-349.

11. Leibowitz R, Do HM, Marcellus ML, et al. Parent vessel occlusion for vertebrobasilar fusiform and dissecting aneurysms. AJNR Am J Neuroradiol. 2003;24(5):902-907.

12. US Food \& Drug Administration. Premarket approval, Pipeline embolization device. US Department of Health \& Human Services. Accessed May 13, 2020. https:// www.accessdata.fda.gov/scripts/cdrh/cfdocs/cfpma/pma. cfm?id=P100018

13. US Food \& Drug Administration. Premarket approval, Pipeline flex embolization device. US Department of Health \& Human Services. Accessed May 13, 2020. https: //www.accessdata.fda.gov/scripts/cdrh/cfdocs/cfpma/pma. cfm?id=P100018S015
14. Bender MT, Colby GP, Jiang B, et al. Flow diversion of posterior circulation cerebral aneurysms: a single-institution series of 59 cases. Neurosurgery. 2019;84(1):206-216.

15. Zammar SG, Buell TJ, Chen CJ, et al. Outcomes after offlabel use of the Pipeline embolization device for intracranial aneurysms: a multicenter cohort study. World Neurosurg. 2018;115:e200-e205.

\section{Disclosures}

Dr. Heit reports being a consultant for MicroVention and Medtronic. Dr. Steinberg reports being a consultant for Qool Therapeutics, Peter Lazic US, NeuroSave, SanBio, Zeiss, and Surgical Theater.

\section{Author Contributions}

Conception and design: Steinberg, Church, Bigder, Sussman, Gummidipundi, Han. Acquisition of data: Steinberg, Church, Bigder, Heit, Do, Dodd, Marks. Analysis and interpretation of data: all authors. Drafting the article: Church. Critically revising the article: all authors. Reviewed submitted version of manuscript: all authors. Approved the final version of the manuscript on behalf of all authors: Steinberg. Statistical analysis: Steinberg, Church, Gummidipundi, Han, Heit. Administrative/technical/ material support: Bigder, Heit, Do. Study supervision: Steinberg, Han, Marks.

\section{Supplemental Information Previous Presentations}

Portions of this work were presented in abstract form at the American Association of Neurological Surgeons Annual Meeting, San Diego, CA, April 13-17, 2019.

\section{Current Affiliations}

Dr. Church: Department of Neurosurgery, Penn State Health, Hershey, PA.

\section{Correspondence}

Gary K. Steinberg: Stanford University School of Medicine, Stanford, CA. gsteinberg@stanford.edu. 